\title{
Low Power All-Digital Radio-over-Fiber Transmission for 28-GHz Band using Parallel Electro-Absorption Modulators
}

\author{
Jakob Declercq, Haolin Li, Joris Van Kerrebrouck, Michiel Verplaetse, Hannes Ramon, \\ Laurens Bogaert, Joris Lambrecht, Chia-Yi Wu, Laurens Breyne, Olivier Caytan, Sam Lemey, \\ Johan Bauwelinck, Xin Yin, Peter Ossieur, Piet Demeester, and Guy Torfs
}

\begin{abstract}
We present a low-power all-digital radio-over-fiber (RoF) transmitter for the $28 \mathrm{GHz}$ band using sigma-delta modulation. Using a parallel electro-absorption modulator (EAM) structure, the radio signal upconversion is split between the electrical and the optical domains. This halves the maximum bandwidth requirement of the driver circuit with respect to conventional implementations. Furthermore, the effect of chromatic dispersion can be mitigated by tuning the optical phase and amplitude applied to the individual modulators, such that transmission notches are partially removed. The modulator structure is described using simplified models and verified in VPI TransmissionMaker. Experimental results using a $140 \mathrm{~mW}$ non-return-to-zero (NRZ) driver and parallel EAMs are provided and yield an error vector magnitude (EVM) of $7.6 \%(5.2 \%)$ when transporting a radio signal modulated at $28 \mathrm{GHz}$ with $5.25 \mathrm{~Gb} / \mathrm{s}(2.625 \mathrm{~Gb} / \mathrm{s})$ 64-QAM over $10 \mathrm{~km}$ standard single mode fiber (SSMF) at $1560 \mathrm{~nm}$.
\end{abstract}

Index Terms-Radio-over-fiber, sigma-delta modulation, mmWave, parallel electro-absorption modulator, chromatic dispersion

\section{INTRODUCTION}

$\mathbf{T}$ HE fifth generation wireless networks (5G) drive research in the direction of massive device connectivity, high data rates and decreased latency. One of the features introduced with $5 \mathrm{G}$ is the deployment of small cells and the utilization of mmWave frequency bands. Cloud radio access networks (C-RANs) in combination with radio-over-fiber (RoF) can be a key-enabling technology to realize this [1]. Three different realizations of RoF links, including digitized RoF (DRoF), analog RoF (ARoF) and sigma-delta-over-fiber (SDoF), have been discussed in prior works [2]-[7]. SDoF simplifies remote radio heads (RRHs) by oversampling the signal and translating it to a two-level signal, combining the benefits of both DRoF (low-cost telecom components) and ARoF (low-complexity RRHs) [2].

This work was supported in part by ERC Advanced Grant ATTO Project under Grant 695495 , in part by the H2020 project 5G-PHOS under Grant 761989 , in part by the UGent Special Research Fund (BOF), and in part by the Research Foundation Flanders (FWO). J. Declercq, H. Li, J. Van Kerrebrouck, M. Verplaetse, H. Ramon, J. Lambrecht, C.-Y. Wu, L. Breyne, O. Caytan, S. Lemey, J. Bauwelinck, X. Yin, P. Ossieur, P. Demeester and G. Torfs are with the Department of Information Technology (INTEC), IDLab, Ghent University-imec, Ghent, 9052 Belgium (e-mail: jakob.declercq@ugent.be; haolin.li@ugent.be; guy.torfs@ugent.be).

L. Breyne and L. Bogaert are with Photonics Research Group, INTEC, Ghent University-imec, Ghent, 9052 Belgium.

J. Declercq and H. Li have equally contributed to this work.
However, the required sample rate will become impractically high for frequency bands above $24 \mathrm{GHz}$ in $5 \mathrm{G}$ New Radio (NR) [8] and the $60 \mathrm{GHz}$ band used by WiGig [9]. To achieve a high sample rate of the sigma-delta modulator (SDM), a low-latency parallelization technique [10] is required and a final multiplexer (MUX) is indispensable to serialize these parallel outputs. A common approach of SDoF transmission uses two low-pass SDMs followed by a digital quadrature upconversion [11], which in practice requires the sampling rate of the final MUX to be 4 times as high as the carrier frequency [12]. The authors of that manuscript have demonstrated a real-time high-speed SDM reaching 100-GS/s covering the 22.75-27.5 GHz band. However, even higher frequency bands become hard to reach with this SDoF approach as state-ofthe-art MUXs can no longer provide the required sample rate. Furthermore, the performance degradation due to jitter and duty-cycle distortions becomes more severe with increasing sample rate, especially when the signaling rates of the MUXs approach the bandwidth limits achievable within a given semiconductor process [12]. Moreover, the required high sample rate also poses a strong limitation on the subsequent driver and $\mathrm{E} / \mathrm{O}$ converter as subsequent stages can hardly provide the required bandwidth to enable the all-digital RoF transmission. To lower the sampling rate required for SDoF transmission of a mmWave radio signal, the authors of [13] modulate the radio signal on an intermediate frequency (IF) and use clock and data recovery (CDR) to extract a local oscillator (LO) frequency for upconversion to the final radio frequency (RF) at the RRH. Although the sample rate of the sigma-delta modulation only amounts to $10 \mathrm{GS} / \mathrm{s}$, additional complexity (clock extraction, mixing) is added to the RRH, decreasing cost-effectiveness.

This work aims to lower the sample rate of the transmitter without adding any complexity at the receiver. To this end, we adopt two low-pass SDMs but the digital quadrature upconversion of I/Q signals is split into two parts: two separate MUXs perform the digital upconversion of the in-phase (I) and quadrature $(\mathrm{Q})$ signals, and these upconverted signals are then combined optically. In this way, the required sample rate can be lowered to the Nyquist sample rate of the desired carrier frequency, i.e. only twice the carrier frequency. This lowers the bandwidth requirements in the MUX, driver, and E/O converter. Moreover, this work maintains the all-digital nature of sigma-delta modulation, which provides robustness 


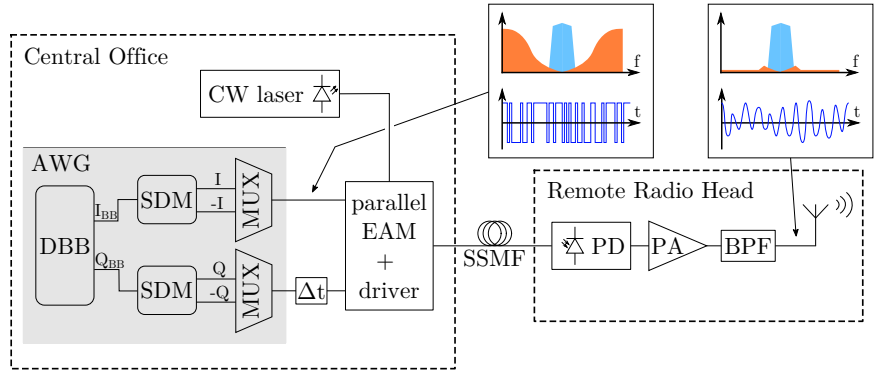

Fig. 1. System architecture of the SDoF downlink connection.

against noise and nonlinear impairments from both optical and microwave transmitter components. An in-house developed differential non-return-to-zero (NRZ) driver IC and parallel electro-absorption modulators (EAMs) are employed to further benefit from the sigma-delta-based all-digital transmission, reducing the footprint and lowering the power consumption. SDoF has the inherent drawback that the quantization noise reduces the modulation efficiency. However, it enables the use of low-power and non-linear components, offsetting this drawback. The parallel EAMs also provide additional flexibility in terms of gain and phase tuning during signal transmission which can be used to compensate the fiber chromatic dispersion to some extent.

This paper is an invited extension of our work presented at OFC 2020 [14], extended with a theoretical analysis of the effects of chromatic dispersion and how they can be compensated with a parallel EAM structure. Section II describes the operational principles of the proposed system in more detail, covering the concepts of sigma-delta modulation, digital upconversion and the use of a parallel EAM structure to combine the I and Q signals optically. The effect of fiber chromatic dispersion is discussed in section III, and a compensation technique is proposed. Furthermore, simulations are performed to indicate the validity of this method, regardless of the chirp parameter of the modulators. In section IV, a detailed description of the experimental setup is given and the associated measurement results are discussed. Finally, a brief conclusion is given in section V.

\section{System Description}

This work considers a downlink SDoF connection between the central office (CO) and a RRH in a C-RAN architecture, transporting an I/Q modulated radio signal at a carrier frequency of $28 \mathrm{GHz}$. Fig. 1 shows the system block diagram. The RRH architecture has a low complexity and solely consists of a photodiode (PD), a power amplifier (PA) and a band-pass filter (BPF), which extracts the radio signal from the detected binary signal. At the central office, the digital baseband (DBB) processing block converts the data to be transmitted into symbols according to a certain constellation and applies pulse shaping. Two parallelized SDMs convert the generated multi-level I and Q signals into two parallel NRZ streams each, representing the even and odd samples. By inverting the odd samples and subsequently interleaving the even and odd streams, digital upconversion is achieved to

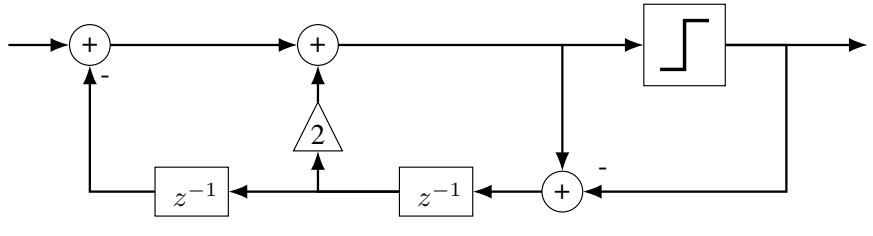

Fig. 2. Block diagram of a second order low-pass SDM [12].

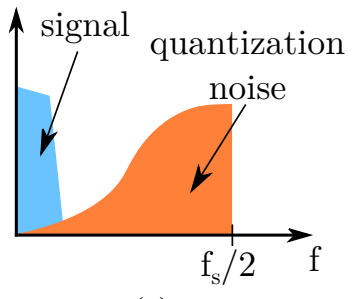

(a)

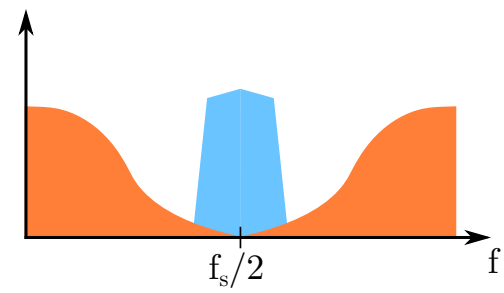

(b)
Fig. 3. Illustration of the output spectrum of a low-pass sigma-delta modulator (left) and of the digital upconversion (right), where $f_{s}=2 f_{c}$.

the Nyquist frequency of the sigma delta streams. In a realtime implementation, the DBB and SDMs can be implemented on a high-end FPGA and the upconversion can be performed by high-speed external multiplexers [12]. However, in this paper, the binary I and Q streams will be generated offline, including the upconversion. An arbitrary waveform generator (AWG) will be used to output these signals at a rate of $56 \mathrm{GS} / \mathrm{s}$. These NRZ streams are then modulated onto the optical carrier and combined in the optical domain by using parallel EAMs. This results in halving the required sample rate with respect to the architecture proposed in [12], where the entire quadrature upconversion happens in the electrical domain.

\section{A. Sigma Delta Modulation and Digital Upconversion}

Fig. 2 shows the block diagram of a second-order low-pass SDM with a single-bit quantizer, which makes it possible to encapsulate a multi-level input signal into a digital bitstream. The binary nature of this stream introduces quantization noise, but due to the oversampling and the feedback inside the modulator, the noise can be shaped to high frequencies, outside of the frequency band in which the signal resides (Fig. 3a). The second-order SDM used in this work exhibits a noise transfer function (NTF) of $\left(1-z^{-1}\right)^{2}$. By inverting every odd bit of the output of a low-pass SDM, the baseband signal is digitally upconverted to a carrier frequency of $f_{c}=f_{s} / 2$, as illustrated in Fig. 3b. The analog RF signal can be extracted from this bitstream by a band-pass filter, eliminating the out-of-band quantization noise.

I/Q modulation can be achieved by applying the oversampled baseband I and Q samples to separate SDMs, after which these signals have to be combined and upconverted to a carrier frequency $f_{c}$. In the analog domain, quadrature upconversion can be accomplished by mixing both signals with quadrature carrier signals. By sampling these quadrature carriers at a rate of $4 f_{c}$, they can be represented by the discrete sequences $[1,0,-1,0]$ and $[0,1,0,-1]$, as is illustrated in Fig. 4. This makes it possible to perform the quadrature upconversion in 


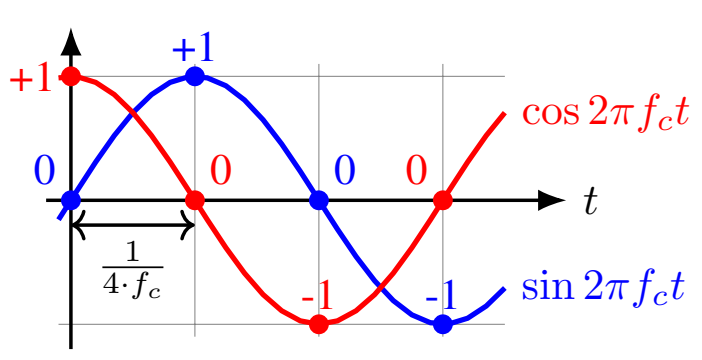

Fig. 4. Sampling the quadrature carrier signals at a sampling rate $f_{s}=4 f_{c}$.

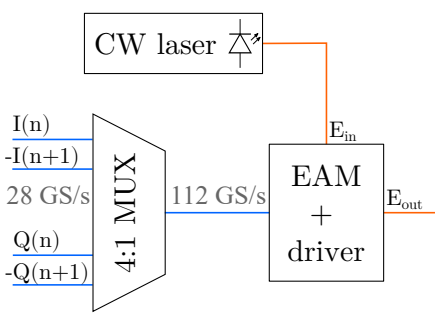

(a)

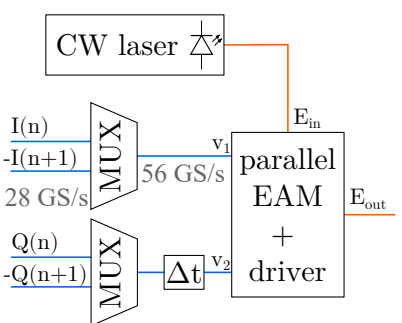

(b)
Fig. 5. Digital quadrature upconversion (a) using a 4-to-1 MUX and a single EAM (b) using two 2-to-1 MUXs and parallel EAMs, as done in this work.

the digital domain by interleaving the I and Q samples in the following way (with $I(n)$ and $Q(n)$ sampled at a rate of $2 f_{c}$ ):

$$
[I(n), Q(n),-I(n+1),-Q(n+1)]
$$

In this way, quadrature upconversion to a carrier of $28 \mathrm{GHz}$ can be achieved by multiplexing four $28 \mathrm{GS} / \mathrm{s}$ datastreams into a single stream of 112 GS/s, as shown in Fig. 5a. However, only state-of-the-art MUXs provide the required sampling rate of $112 \mathrm{GS} / \mathrm{s}$, and reaching even higher frequency bands becomes nearly impossible [12].

Therefore, in this work, the quadrature upconversion is split over the electrical and optical domain. The separate baseband signals are first upconverted in the electrical domain and later added together in the optical domain. As illustrated in Fig. 5b, the I and Q streams coming from the SDMs are upconverted using separate MUXs, operating at a rate of only $2 f_{c}=56 \mathrm{GS} / \mathrm{s}$. The resulting two NRZ streams represent the analog RF signals $I(t) \cos \left(2 \pi f_{c} t\right)$ and $Q(t) \cos \left(2 \pi f_{c} t\right)$. In order to obtain the quadrature signal $Q(t) \sin \left(2 \pi f_{c} t\right)$, a delay of $\Delta t=\frac{1}{4 f_{c}}$ is applied to the $\mathrm{Q}$ stream, which introduces a phase shift of $\pi / 2$ at the carrier frequency. Although this delay causes an I/Q mismatch, it can be neglected when the signal bandwidth of interest is small w.r.t. the carrier frequency. For broader signals, the I/Q mismatch can be easily precompensated in the digital baseband [12].

\section{B. Optical combination of I and $Q$ signals}

To combine the upconverted I and Q signals, a parallel EAM topology is adopted. A block diagram is shown in Fig. 6. In this structure, the optical carrier is split into two branches, which are modulated by the upconverted I and Q signals using EAMs. Both optical signals are combined at the output using a tunable power combiner, yielding the desired optical signal, modulated by the coherent electrical bandpass signal.

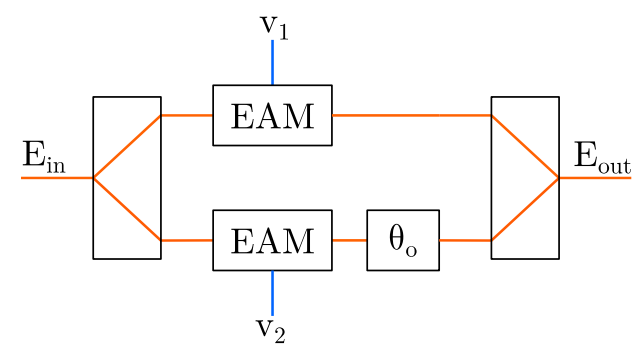

Fig. 6. Block diagram showing the structure of parallel EAMs.

By tuning the combiner ratio, variations in modulation depth of both EAMs can be mitigated.

\section{INFLUENCE OF Chromatic Dispersion}

As mentioned before, next to the reduced sample rates of the NRZ streams, there is another advantage to using this SDoF transmitter architecture: by tuning the value $\theta_{o}$ of the optical phase shifter that is present in one of the arms of the parallel EAM structure, it is possible to reduce the effects of chromatic dispersion in the optical fiber.

Chromatic dispersion introduces frequency-dependent loss in intensity-modulation direct-detection links. Around $5 \mathrm{~km}$ of transmission in C-band over a SSMF, the chromatic dispersion notch aligns with the $28 \mathrm{GHz}$ frequency band [12], limiting the reach of low-cost mmWave RoF links. Due to chromatic dispersion, the different frequency components travel at a different speed, causing a phase difference between the side bands and the carrier of an intensity modulated signal, given by

$$
\Delta \phi=-L \frac{\lambda_{o}}{2 \omega_{o}} D \omega_{c}^{2}
$$

with $\lambda_{o}$ and $\omega_{o}$ respectively the wavelength and pulsation of the optical carrier, L the fiber length and D the dispersion parameter, which is around $16 \mathrm{ps} / \mathrm{nm} / \mathrm{km}$ at $1550 \mathrm{~nm}$ in SSMF and $\omega_{c}$ the RF carrier pulsation in the electrical domain [15].

\section{A. Compensation of Chromatic Dispersion}

The effect of this phase difference can be mitigated using the parallel EAMs (Fig. 6). The structure can be modeled in first approximation with the following linearized model (neglecting the modulator chirp):

$$
E_{\text {out }}=\frac{A}{2} e^{j \omega_{o} t}\left(v_{1}(t)+v_{2}(t) e^{j \theta_{o}}\right)
$$

Notice that the EAMs will be used to transport a sigma-delta modulated signal. Hence, they will be driven by NRZ signals, justifying the linearized model.

At the input of the EAMs, the upconverted sigma-delta modulated I and Q signals are applied together with a DC bias. These are represented by the signals $v_{1}(t)=I \cos \left(\omega_{c} t\right)+V_{b}$ and $v_{2}(t)=Q \sin \left(\omega_{c} t\right)+V_{b}$. When these signals are applied 


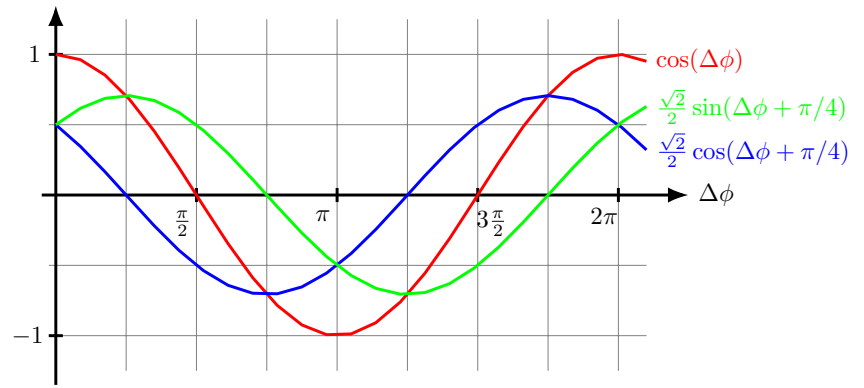

Fig. 7. Curves indicating the performance for the two analyzed cases: $\theta_{o}=0$ (red) and $\theta_{o}=-\pi / 2$ (blue and green).

to the parallel EAMs, and are transported over a length of fiber, the optical field can be represented as follows:

$$
\begin{gathered}
E_{\text {out }}=\frac{A}{2}\left(\frac{I+Q e^{j\left(\theta_{o}-\pi / 2\right)}}{2} e^{j\left(\left(\omega_{o}+\omega_{c}\right) t+\Delta \phi\right)}\right. \\
+\frac{I-Q e^{j\left(\theta_{o}-\pi / 2\right)}}{2} e^{j\left(\left(\omega_{o}-\omega_{c}\right) t+\Delta \phi\right)} \\
\left.+2 \cos \left(\theta_{o} / 2\right) V_{b} e^{j\left(\omega_{o} t+\theta_{o} / 2\right)}\right)
\end{gathered}
$$

The two sidebands have undergone an additional phase shift of $\Delta \phi$ w.r.t. the carrier due to chromatic dispersion. The photodiode at the RRU detects the optical power, proportional to $\left|E_{\text {out }}\right|^{2}$, which contains the following signal in band:

$$
\begin{aligned}
|A|^{2} V_{b} \cos \left(\theta_{o} / 2\right)(I & \cos \left(\omega_{c} t\right) \cos \left(\theta_{o} / 2-\Delta \phi\right) \\
& \left.+Q \sin \left(\omega_{c} t\right) \cos \left(\theta_{o} / 2+\Delta \phi\right)\right)
\end{aligned}
$$

At fiber lengths where $\Delta \phi \approx k \pi, k \in \mathbb{Z}$, it is optimal to choose $\theta_{o}=0$. In that case (5) reduces to (6) and contains the desired radio signal:

$$
|A|^{2} V_{b} \cos (\Delta \phi)\left(I \cos \left(\omega_{c} t\right)+Q \sin \left(\omega_{c} t\right)\right)
$$

It is obvious that at fiber lengths where $\Delta \phi \approx \pi / 2+k \pi, k \in \mathbb{Z}$, the received signal power is heavily reduced. This is similar to the behavior of typical optical double sideband modulation [16]. However, the signal can be recovered by changing $\theta_{0}$ to a value of $-\pi / 2$. Then (5) reduces to (7):

$$
\begin{aligned}
|A|^{2} V_{b} \frac{\sqrt{2}}{2}(I & \cos \left(\omega_{c} t\right) \cos (\Delta \phi+\pi / 4) \\
& \left.+Q \sin \left(\omega_{c} t\right) \sin (\Delta \phi+\pi / 4)\right)
\end{aligned}
$$

Now, the I and $\mathrm{Q}$ signals are scaled by a different factor. For $\Delta \phi=\pi / 2$ the $\mathrm{I}$ and $\mathrm{Q}$ signal are both scaled with a factor $1 / 2$ w.r.t to the optical back-to-back (B2B) transmission shown in (6). Besides, the sign of the in-phase component is flipped. This should be compensated at the input of the modulator by inverting the input signal. These factors that scale the I/Q signals, along with the factor $\cos (\Delta \phi)$ of (6) are plotted in Fig. 7. For other values of $\Delta \phi$ than those discussed previously, optimal results are achieved for different values of $\theta_{o}$. Note that in these other cases, a gain imbalance between the I and Q signals degrades the performance. It is possible to

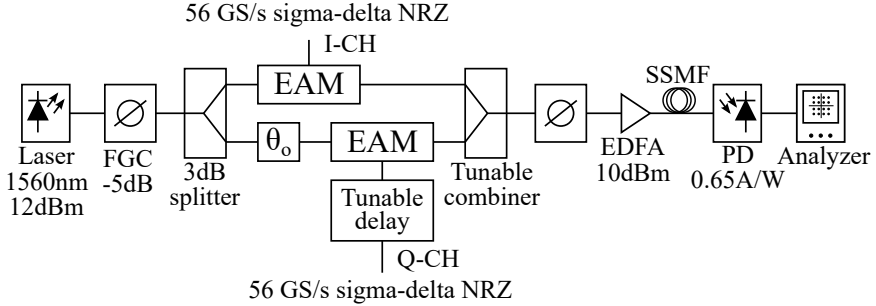

Fig. 8. Schematic representation of the model used to simulate the transmitter.

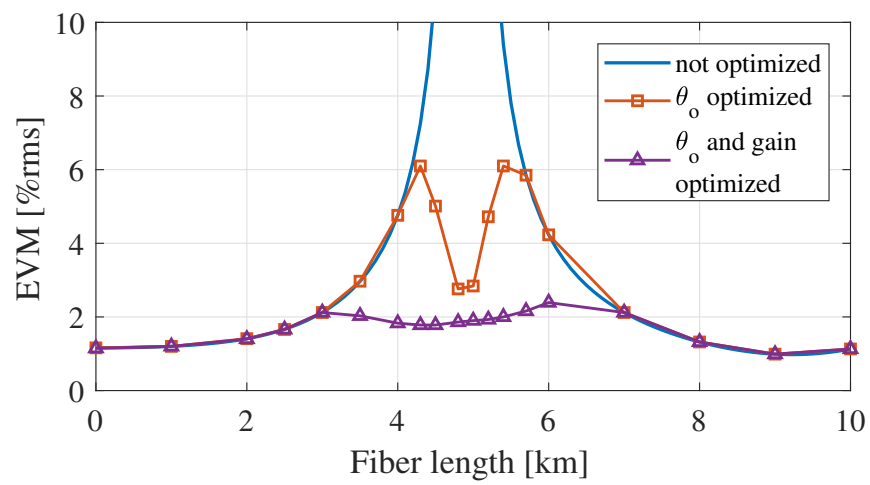

Fig. 9. Simulated performance of the parallel EAM system in the case of chirp-free modulators, for different fiber lengths.

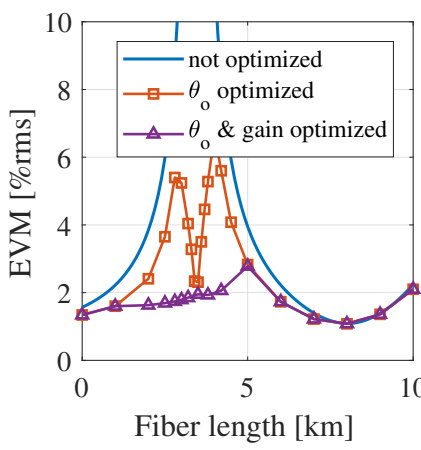

(a) $\alpha_{e}=0.5$

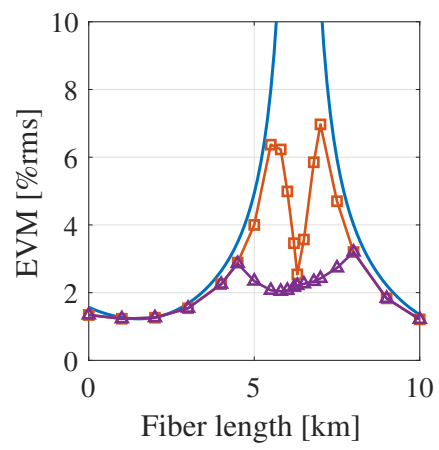

(b) $\alpha_{e}=-0.5$
Fig. 10. Simulated performance of the parallel EAM system for different fiber lengths when the EAMs exhibit chirp.

partially compensate for this imbalance at the transmitter by adjusting the tunable optical power combiner of the parallel EAM structure. The remainder can be canceled by altering the output swing of the driver IC or by scaling the baseband I and Q signals.

\section{B. Verification in VPI TransmissionMaker}

In order to assess the performance of the proposed system, simulations are performed using VPIphotonics Transmissionmaker ${ }^{\mathrm{TM}}$. The in-phase and quadrature components of a 437.5 MBd QPSK symbol stream are applied to second-order SDMs and subsequently digitally upconverted at a rate of $56 \mathrm{GS} / \mathrm{s}$, with an associated carrier frequency of $f_{c}=28 \mathrm{GHz}$. The resulting NRZ streams are applied to the transmitter architecture shown in figure 8 . To focus the analysis on the effect of chromatic dispersion, the attenuation of the fiber 


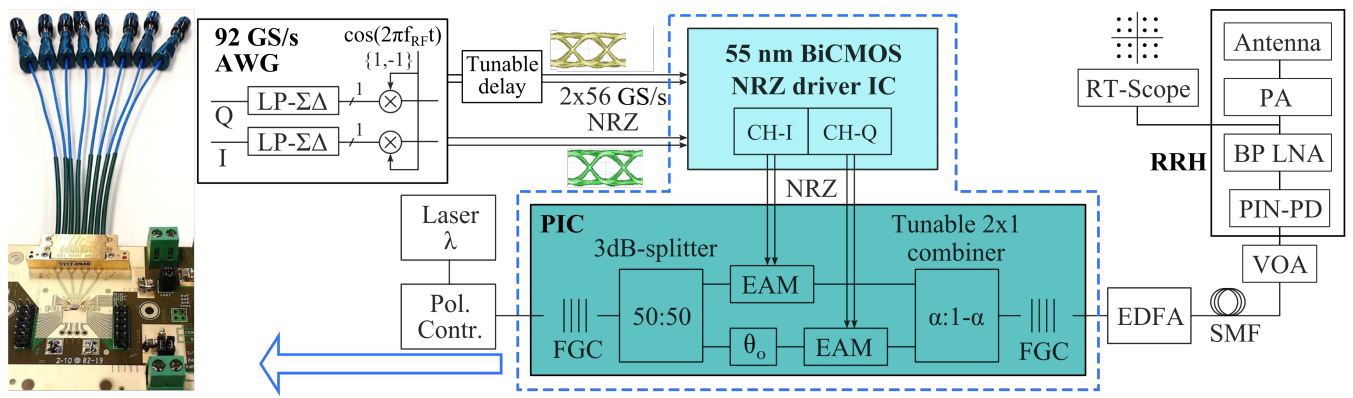

Fig. 11. Block diagram and experimental setup for the sigma-delta radio-over-fiber transmission using parallel EAMs.

is not considered and noise sources are not modeled. In the experimental setup, an erbium-doped optical amplifier (EDFA) is included to increase the signal-to-noise ratio (SNR). Alternatively, specialized narrow-band receivers such as [17] will significantly lower the noise contributions.

If the optical phase shift were to be kept at $\theta_{o}=0$, the received signal power would be reduced drastically at fiber lengths where $\Delta \phi \approx \pi / 2+k \pi, k \in \mathbb{Z}$, resulting in very high error vector magnitude (EVM) values. For a carrier frequency of $f_{c}=28 \mathrm{GHz}$, a dispersion coefficient of $D=16 \mathrm{ps} / \mathrm{nm} / \mathrm{km}$ and a laser wavelength of $\lambda_{o}=1560 \mathrm{~nm}$, this occurs at fiber lengths of $L=4.91 \mathrm{~km}, 14.7 \mathrm{~km}, 24.6 \mathrm{~km}$, etc (as in the nonoptimized case of Fig. 9).

However, by tuning the optical phase shift $\theta_{o}$, it is possible to minimize the EVM of the received symbols for all possible fiber lengths. This is supported by the simulation results of Fig. 9, where the EVM values remain well below $8 \%$ rms. Still, at fiber lengths around $4 \mathrm{~km}$ and $6 \mathrm{~km}$, a gain imbalance between the received I and Q signals causes an increase of the EVM compared to EVM values at other fiber lengths. By tuning the amplitude of the NRZ signals applied to the EAMs, this gain imbalance can be pre-compensated, yielding the lowest curve of Fig. 9, where only a minor dependency of the received signal quality to the fiber length is observed.

In the previous analysis, the modulators were supposed to be chirp-free, which is generally not the case. The exact chirp parameter $\alpha_{e}$ of the used EAMs is not known. To verify the operation of the proposed system for different chirp parameters, the simulations have been repeated with $\alpha_{e}= \pm 0.5$. The results of these simulations are shown in Fig. 10 and indicate that the effect of this chirp can also be compensated by tuning the value of $\theta_{0}$. Note that, due to the periodic nature of the chromatic dispersion effect, the characteristics of Fig. 9 and 10 continue periodically for fiber lengths greater than $10 \mathrm{~km}$.

\section{EXPERIMENTAL RESUlts}

\section{A. Experimental setup}

The system architecture and the corresponding experimental setup are illustrated in Fig. 11. First, the I and Q components of a baseband QAM-signal (pulse-shaped with a square-rootraised-cosine filter with roll-off 0.28 ) are oversampled and noise-shaped by second-order sigma-delta modulators, which are implemented off-line, at an equivalent sample rate of
56 GS/s. The generated NRZ streams of both I and Q channels are further digitally upconverted to a carrier frequency $f_{c}=28 \mathrm{GHz}$ by toggling the bits at odd clock cycles, which generates two NRZ streams representing the analog RF signals $I(t) \cos \left(2 \pi f_{c} t\right)$ and $Q(t) \cos \left(2 \pi f_{c} t\right)$. A $92 \mathrm{GS} / \mathrm{s}$ arbitrary waveform generator (AWG) from Keysight is used to output these two NRZ streams with an amplitude of $200 \mathrm{mVpp}$ at a rate of $56 \mathrm{GS} / \mathrm{s}$. The $\mathrm{Q}$ stream goes through an additional delay line, which introduces a phase shift of $\pi / 2$ at the carrier frequency in order to obtain the quadrature signal $Q(t) \sin \left(2 \pi f_{c} t\right)$. An accurate clock (only 150 fs rms jitter) was used as a reference for the generation of both $56 \mathrm{GS} / \mathrm{s}$ streams at the AWG, maintaining tight synchronization between these signals and ensuring as little degradation due to jitter as possible [18], [19].

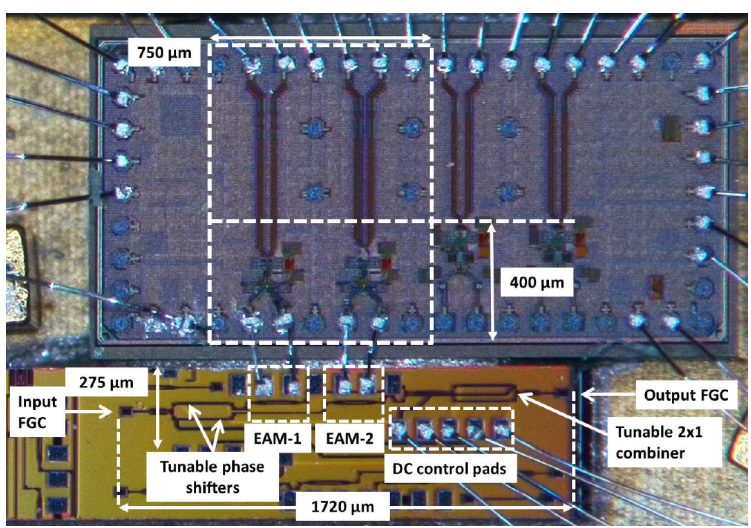

Fig. 12. Si-integrated transmitter, requiring $0.275 \mathrm{~mm}$ by $1.72 \mathrm{~mm}$ on PIC and $0.4 \mathrm{~mm}$ by $0.75 \mathrm{~mm}$ on EIC, including heaters, bondpads and couplers [20].

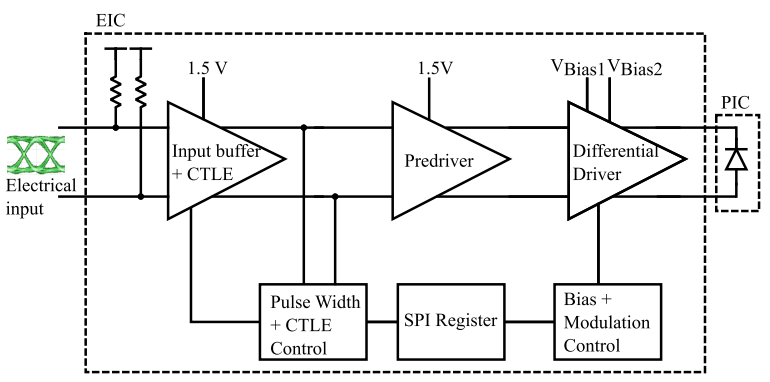

Fig. 13. Block diagram of one channel of the BiCMOS EAM driver IC, which biases and drives the EAMs differentially. EIC: electrical integrated circuit. PIC: photonic integrated circuit. CTLE: continuous-time linear equalizer. 

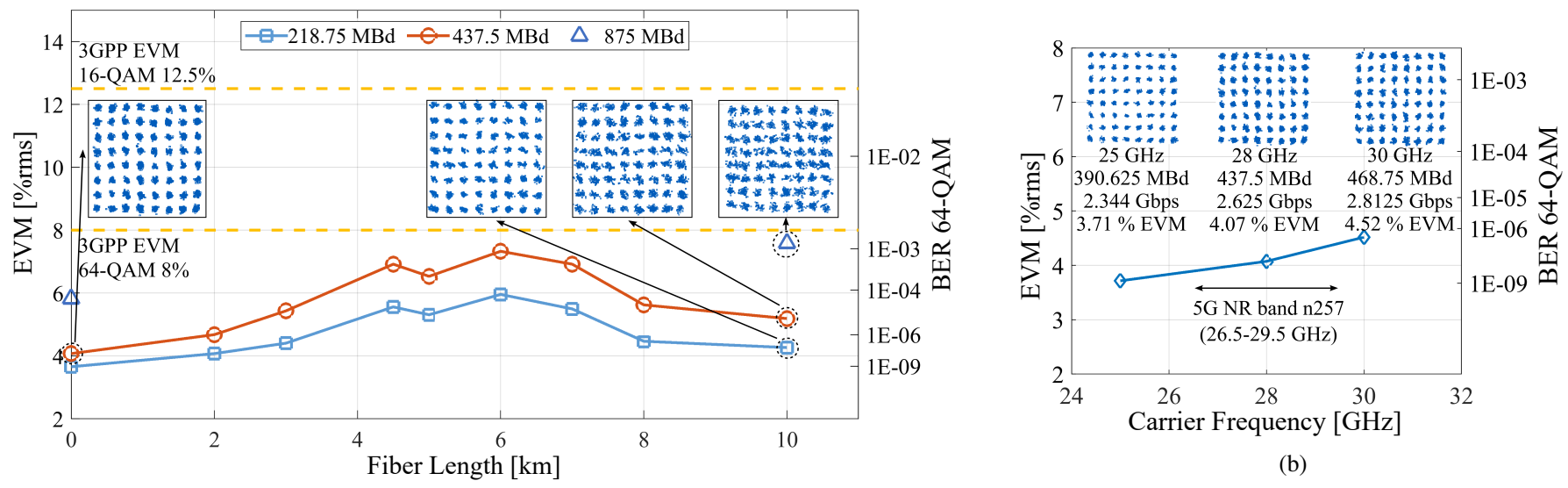

(a)

Fig. 14. (a) Measured EVM vs. fiber spans at $28 \mathrm{GHz}$ carrier frequency. Demodulated constellations are indicated for optical B2B and $10 \mathrm{~km}$ of fiber. (b) Measured EVM and demodulated constellation vs. different carrier frequencies in optical B2B.

The NRZ signals are subsequently sent through a 6-inch $50 \mathrm{GHz}$ multi-coax connector assembly towards two channels of an EAM driver IC, which is wirebonded to the parallel EAMs (Fig. 12) and to the PCB hosting both the electrical and optical chips. As shown in Fig. 13, the non-linear EAM driver IC amplifies the input NRZ signals, reversely biases the EAMs and drives them differentially with approximately $2 \mathrm{Vpp}$. This in-house developed IC is fabricated in a $55 \mathrm{~nm}$ SiGe BiCMOS technology [21] and consumes approximately $140 \mathrm{~mW}$ for both channels together, including the biasing of the EAMs. The modulators are compact GeSi EAMs with a bandwidth of over $50 \mathrm{GHz}$, fabricated on a silicon photonics platform [22]. Their chirp factor was measured to be very low (around -0.01 ) at the wavelength and bias voltage used in this work. A $1560 \mathrm{~nm} 12 \mathrm{dBm}$ external laser is coupled into the PIC through fiber-to-grating couplers (FGCs) with approximately $5 \mathrm{~dB}$ loss per coupler. By adjusting the thermally tunable optical power combiner, a potential gain mismatch between the outputs of both EAMs can be resolved. Finally, the optical output power of this transmitter is boosted to $10 \mathrm{dBm}$ by means of an EDFA.

A single-mode fiber carries the optical signal to the RRU, where a photodiode detects the modulated signal. The signal power arriving at this commercial $40 \mathrm{GHz}$ III-V-based $50 \Omega$ PIN-PD (Finisar XPDV2120RA, with a responsivity of $0.65 \mathrm{~A} / \mathrm{W}$ ) is controlled by a variable optical attenuator (VOA). In order to remove the out-of-band quantization noise and amplify the signal of interest, a matched narrowband receiver, such as the one presented in [17] could be used. However, in this work, this is realized by a commercial band-pass low noise amplifier (LNA). In a real application, the RF signals would be subsequently applied to a power amplifier and an antenna, completing the RoF downlink transmission. This aspect is not considered here, but a Keysight real-time oscilloscope (DSAZ634A) and VSA software are used to demodulate the RF signal and evaluate the performance of the system.

\section{B. Measurement results}

The signal quality obtained with the proposed test setup was investigated by measuring the EVM, referenced to the root-mean-square (rms) amplitude of the signal. To explore the reach of the SDoF transmission, the EVM values were measured for different fiber lengths and compared in Fig. 14a. The measured EVMs (at a fixed received optical power of $4 \mathrm{dBm}$ at the PIN-PD) are $3.66 \%$ and $4.07 \%$ for $278.25 \mathrm{MBd}$ and 437.5 MBd 64-QAM in optical B2B. These EVMs enable adequate signal quality for 64-QAM with $1 \mathrm{E}-05$ bit error rates (BERs) before error correction [23], and can be mainly attributed to the Relative Intensity Noise (RIN) of the laser, which is further increased by the EDFA [24]. The noise could be reduced by increasing the laser power, using a laser with a lower RIN, and replacing the grating couplers by edge couplers (which exhibit lower losses). Below $3 \mathrm{~km}$, chromatic dispersion has little influence.

At larger reaches, the optical phase shift $\theta_{o}$ and the gain of the optical power combiner are tuned in order to compensate the effect of chromatic dispersion. However, this reduces the power at the input of the EDFA, leading to increased amounts of noise and thus higher EVM values. At a fiber length of $6 \mathrm{~km}$, the optical power is reduced by $3 \mathrm{~dB}$ (see eq. 7), corresponding to a gain reduction of $6 \mathrm{~dB}$ in comparison to the $\mathrm{B} 2 \mathrm{~B}$ link. In the absence of other noise sources, this would increase the overall noise figure (NF) by $6 \mathrm{~dB}$ and the EVM would increase by a factor 2 . However, due to the contribution of the noise generated at the EDFA and the receiver, the NF increase is smaller $(4.3 \mathrm{~dB}$ and $5 \mathrm{~dB}$ for the $218.75 \mathrm{MB}$ and 437.5 MB signals respectively). For a fiber length of $10 \mathrm{~km}$, no compensation is required, leading again to a better EVM. In that case, a small degradation in EVM is observed in comparison to optical $\mathrm{B} 2 \mathrm{~B}$, but this can be attributed to an increased optical insertion loss.

The total bitrate of the $437.5 \mathrm{MBd}$ 64-QAM signal is $2.625 \mathrm{~Gb} / \mathrm{s}$ and is doubled to $5.25 \mathrm{~Gb} / \mathrm{s}$ when transmitting an $875 \mathrm{MBd}$ 64-QAM signal, halving the amount of oversampling. However, in that case, apart from the measurements at a fiber length of $0 \mathrm{~km}$ and $10 \mathrm{~km}$, the measured EVM values were too high and are not reported. As shown in Fig. 14b, this all-digital RoF transmitter targets the 5G NR band $\mathrm{n} 257$ specified in 3GPP [8] and measured EVMs satisfy the 3GPP 
requirement for 64-QAM: EVM $<8 \%$ [25]. By limiting the noise generated at the laser and EDFA, further research might meet the 256-QAM and even 1024-QAM 5G-NR standards.

The total power consumption of this SDoF transmitter is only around $180 \mathrm{~mW}$ (excluding laser and AWG, but including on-chip thermo-optic phase shifters), by avoiding components such as high-speed DACs and frequency upconverters. The transmitter only requires two $200 \mathrm{mVpp}$ NRZ inputs. The highspeed sigma-delta modulator and its parallelization technique from [12] can be employed to replace the AWG. Due to the very compact footprint of the EAMs and NRZ driver IC, it would be feasible to integrate them together with the sigmadelta modulator and MUX into a single module, fitting a standard pluggable form factor such as QSFP.

By using the high-speed MUXs at twice the sample rate (112 GS/s) [26], it would theoretically be possible to double the carrier frequency of the transmitted RF signal to $56 \mathrm{GHz}$. However, this is not possible using the current setup due to limitations on the speed of the EAM driver. Alternatively, the carrier frequency could be increased by adopting a different SDoF architecture where an IF signal is transported via SDoF and further upconversion happens at the RRU, at the expense of increased RRU complexity [13].

\section{CONCLUSION}

We demonstrated a low-power Si-integrated all-digital radio-over-fiber transmitter for operation in the $28 \mathrm{GHz}$ band and beyond by using sigma-delta modulation and parallel EAMs. By splitting the digital quadrature upconversion, part of it is performed in the optical domain. This transmitter architecture halves the required sample rate and bandwidth of the used microwave and photonics components compared to prior works, while maintaining the digital nature of sigmadelta modulation. In addition, the effect of chromatic dispersion can be compensated to some extent, meaning that the operation of this transmitter is guaranteed, regardless of the fiber length. The prominent performance $(5.25 \mathrm{~Gb} / \mathrm{s}$ 64-QAM transmission at a carrier frequency of $28 \mathrm{GHz}$ over a fiber length of more than $10 \mathrm{~km}$, accompanied by a wide carrier frequency coverage) corroborates the strong competitiveness of this SDoF approach in high-frequency band radio-over-fiber communications. At the time of writing, it is the first time to realize EAM-based all-digital radio-over-fiber transmission at carrier frequencies beyond $28 \mathrm{GHz}$.

\section{ACKNOWLEDGMENT}

The authors would like to thank VPIphotonics for the academic license.

\section{REFERENCES}

[1] C. Ranaweera, E. Wong et al., "5G C-RAN with optical fronthaul: An analysis from a deployment perspective," Journal of Lightwave Technology, vol. 36, no. 11, pp. 2059-2068, 2018.

[2] L. Breyne, G. Torfs et al., "Comparison between analog radio-over-fiber and sigma delta modulated radio-over-fiber," IEEE Photonics Technology Letters, vol. 29, no. 21, pp. 1808-1811, 2017.

[3] C.-Y. Wu, H. Li et al., "Real-time 4x3.5 Gbps sigma delta radio-overfiber for a low-cost 5G C-RAN downlink," in European Conference on Optical Communication (ECOC), Sep. 2018, pp. 1-3.
[4] J. Wang, Z. Jia et al., "Delta-sigma modulation for next generation fronthaul interface," Journal of Lightwave Technology, vol. 37, no. 12, pp. 2838-2850, 2019.

[5] N. Argyris, G. Giannoulis et al., "A 5G mmwave fiber-wireless IFoF analog mobile fronthaul link with up to $24-\mathrm{Gb} / \mathrm{s}$ multiband wireless capacity," Journal of Lightwave Technology, vol. 37, no. 12, pp. 2883 2891, 2019.

[6] M. Sung, S. Cho et al., "Demonstration of IFoF-based mobile fronthaul in $5 \mathrm{G}$ prototype with $28-\mathrm{GHz}$ millimeter wave," Journal of Lightwave Technology, vol. 36, no. 2, pp. 601-609, 2018.

[7] L. Bogaert, J. V. Kerrebrouck et al., "SiPhotonics/GaAs 28-GHz transceiver for mmwave-over-fiber laser-less active antenna units," in Optical Fiber Communication Conference Postdeadline Papers, Mar. 2020, p. Th4A.5.

[8] “3GPP Release 15 Overview." [Online]. Available: https://spectrum.ieee.org/telecom/wireless/3gpp-release-15-overview

[9] L. Verma, M. Fakharzadeh, and S. Choi, "Wifi on steroids: 802.11 AC and 802.11AD," IEEE Wireless Communications, vol. 20, no. 6, pp. $30-35,2013$.

[10] H. Li, L. Breyne et al., "A 21-GS/s single-bit second-order delta-sigma modulator for FPGAs," IEEE Transactions on Circuits and Systems II: Express Briefs, vol. 66, no. 3, pp. 482-486, 2019.

[11] A. Frappe, A. Flament et al., "An all-digital RF signal generator using high-speed $\Delta \Sigma$ modulators," IEEE Journal of Solid-State Circuits, vol. 44, no. 10, pp. 2722-2732, 2009.

[12] H. Li, M. Verplaetse et al., "Real-time 100-GS/s sigma-delta modulator for all-digital radio-over-fiber transmission," Journal of Lightwave Technology, vol. 38, no. 2, pp. 386-393, 2020.

[13] C. Wu, H. Li et al., "Distributed antenna system using sigma-delta intermediate-frequency-over-fiber for frequency bands above $24 \mathrm{GHz}$," Journal of Lightwave Technology, vol. 38, no. 10, pp. 2765-2773, 2020.

[14] H. Li, J. V. Kerrebrouck et al., "Low power all-digital radio-overfiber transmission for $28-\mathrm{GHz}$ band using parallel electro-absorption modulators," in Optical Fiber Communication Conference (OFC), Mar. 2020, p. M2F.6.

[15] G. Meslener, "Chromatic dispersion induced distortion of modulated monochromatic light employing direct detection," IEEE Journal of Quantum Electronics, vol. 20, no. 10, pp. 1208-1216, 1984.

[16] G. H. Smith, D. Novak, and Z. Ahmed, "Overcoming chromaticdispersion effects in fiber-wireless systems incorporating external modulators," IEEE Transactions on Microwave Theory and Techniques, vol. 45, no. 8, pp. 1410-1415, 1997.

[17] L. Bogaert, H. Li et al., "36 Gb/s narrowband photoreceiver for mmwave analog radio-over-fiber," Journal of Lightwave Technology, vol. 38 , no. 12 , pp. 3289-3295, 2020.

[18] S. Jang, B. Park, and S. Hong, "Digital radio-over-fiber system with multi-pulse manchester encoding-assisted delta-sigma modulation," Opt. Express, vol. 25, no. 7, pp. 8335-8349, Apr 2017. [Online]. Available: http://www.opticsexpress.org/abstract.cfm?URI=oe-25-7-8335

[19] S. Jang, S. Kong et al., "Impact of non-idealities on the performance of delta-sigma/manchester-modulated microwave signals," in 2019 49th European Microwave Conference (EuMC), 2019, pp. 1040-1043.

[20] J. Lambrecht, J. Verbist et al., "Low-power (1.5 pJ/b) silicon integrated 106 Gb/s PAM-4 optical transmitter," Journal of Lightwave Technology, vol. 38 , no. 2 , pp. 432-438, 2020 .

[21] H. Ramon, J. Lambrecht et al., "70 Gb/s low-power dc-coupled NRZ differential electro-absorption modulator driver in $55 \mathrm{~nm}$ SiGe BiCMOS," Journal of Lightwave Technology, vol. 37, no. 5, pp. 1504-1514, 2019.

[22] S. A. Srinivasan, P. Verheyen et al., "50Gb/s C-band GeSi waveguide electro-absorption modulator," in 2016 Optical Fiber Communications Conference and Exhibition (OFC), 2016, pp. 1-3.

[23] R. A. Shafik, M. S. Rahman, and A. R. Islam, "On the extended relationships among EVM, BER and SNR as performance metrics," in International Conference on Electrical and Computer Engineering, 2006, pp. 408-411.

[24] A. W. Setiawan Putra, M. Yamada et al., "Theoretical analysis of noise in erbium doped fiber amplifier," IEEE Journal of Quantum Electronics, vol. 53, no. 4, pp. 1-8, 2017.

[25] 3GPP TS 36.104, "Evolved universal terrestrial radio access (E-UTRA): Base station (BS) radio transmission and reception (Release 16)," V16.5.0, Mar. 2020.

[26] J. Verbist, M. Verplaetse et al., "Real-time $100 \mathrm{~Gb} / \mathrm{s}$ NRZ and EDB transmission with a GeSi electroabsorption modulator for short-reach optical interconnects," Journal of Lightwave Technology, vol. 36, no. 1, pp. 90-96, 2018. 